\title{
Correction to: Transmission risk
} beyond the village: entomological and human factors contributing to residual malaria transmission in an area approaching malaria
elimination on the Thailand-Myanmar border

Hannah M. Edwards ${ }^{1,2^{*}} \mathbb{0}$, Patchara Sriwichai ${ }^{3}$, Kirakorn Kirabittir ${ }^{4}$, Jetsumon Sattabongkot ${ }^{4}$, Irwin F. Chavez ${ }^{5}$ and Jeffrey $\mathrm{Hii}^{1}$

\section{Correction to: Malar J (2019) 18:221}

https://doi.org/10.1186/s12936-019-2852-5

Following publication of the original article [1], the authors advised of two errors present in the article: one concerning two author names and the other missing funding details.

The errors are:

1. The named authors of 'Jetsumon Prachumsri' and 'Kirakorn Kirabittir' are incorrect and should read respectively as:

'Jetsumon Sattabongkot' and 'Kirakorn Kiattibutr'

2. Under the 'Study area' sub-section of the 'Methods' section, this sentence is missing funding details:

"As part of a concurrent longitudinal study in the area, prevalence by microscopy in the three villages was found to be $0.71 \%, 0.89 \%$ and $0.27 \%$ in January, May and November 2016, respectively (Fig. 1); all infections were caused by Plasmodium vivax."

However, the sentence should read as:

"As part of a concurrent longitudinal study in the area from Mahidol University (ICEMR and D43 funded Projects U19AI0819672 and D43TW006571), preva-

*Correspondence: hannah.edwards11@imperial.ac.uk

${ }^{1}$ Department of Disease Epidemiology, Imperial College London, London, UK

Full list of author information is available at the end of the article lence by microscopy in the three villages was found to be $0.71 \%, 0.89 \%$ and $0.27 \%$ in January, May and November 2016, respectively (Fig. 1); all infections were caused by Plasmodium vivax."

The authors apologize for these errors.
Author details

${ }^{1}$ Department of Disease Epidemiology, Imperial College London, London, UK. ${ }^{2}$ Malaria Consortium Asia, Faculty of Tropical Medicine, Mahidol University, 420/6 Rajvithi Road, Bangkok 10400, Thailand. ${ }^{3}$ Department of Entomology, Faculty of Tropical Medicine, Mahidol University, 420/6 Rajvithi Road, Bangkok 10400, Thailand. ${ }^{4}$ Mahidol Vivax Research Unit, Faculty of Tropical Medicine, Mahidol University, 420/6 Rajvithi Road, Bangkok 10400, Thailand. ${ }^{5}$ Department of Tropical Hygiene, Faculty of Tropical Medicine, Mahidol University, 420/6 Rajvithi Road, Bangkok 10400, Thailand.

The original article can be found online at https://doi.org/10.1186/s1293 6-019-2852-5.

Published online: 24 July 2019

\section{Reference}

1. Edwards HM, Sriwichai P, Kirabittir K, Prachumsri J, Chavez IF, Hii J. Transmission risk beyond the village: entomological and human factors contributing to residual malaria transmission in an area approaching malaria elimination on the Thailand-Myanmar border. Malar J. 2019;18:221. https ://doi.org/10.1186/s12936-019-2852-5.

\section{Publisher's Note}

Springer Nature remains neutral with regard to jurisdictional claims in published maps and institutional affiliations. 